\title{
Detection of Germline Mutations in Argentine Retinoblastoma Patients: Low and Full Penetrance Retinoblastoma Caused by the Same Germline Truncating Mutation
}

\author{
Viviana Dalamón*, Ezequiel Surace , Florencia Giliberto, Verónica Ferreiro, \\ Cecilia Fernandez and Irene Szijan \\ Cátedra de Genética y Biología Molecular, Facultad de Farmacia y Bioquímica, Universidad de Buenos Aires, Argentina \\ ${ }^{\S}$ Department of Neurology, Washington University School of Medicine, St. Louis, MO 63110, USA
}

Received 9 June 2003, Accepted 30 July 2003

\begin{abstract}
Constitutional RB1 gene mutations were studied in a series of 21 families with unilateral and bilateral retinoblastoma patients. Peripheral blood lymphocytes were analyzed by "exon by exon" PCR-heteroduplex and sequencing. Mutations were identified in 6 (29\%) of the patients. One mutation corresponded to an intronic polymorphism in g.174351T > A. The other five mutations resulted $\mathrm{C}$ to $\mathrm{T}$ exonic transitions, four were CGA sequences (g.65386, g.150037 in two patients, and g.162237), creating stop codons and presumably truncated proteins. The fifth one was new and resulted in alanine to valine substitution (g.73774). Two patients had the same the germline truncated mutation (g.150037C $>\mathrm{T}$ ), one with a familial bilateral early onset retinoblastoma and one with a sporadic unilateral late onset retinoblastoma. The later type has not been previously described. This finding is discussed in the genotype/phenotype correlation context. Additionally, a single nucleotide change was found in six studied samples, where a $\mathrm{C}$ to $\mathrm{T}$ homozygous transversion was identified in intron 26 (IVS26 + 28). It is worthy the non concordance of the nucleotide with the published sequence. This analysis proved to be a useful method for the detection of mutations in the RB1 gene, and contributed to the adequate genetic counseling to patients and relatives.
\end{abstract}

Keywords: RB1 gene, Retinoblastoma, Germline mutations, $\mathrm{C}$ - to - T transition, Low penetrance

*To whom correspondence should be addressed.

Tel: 5411-5950-8805; Fax: 5411-4964-8296

E-mail:vdalam@ffyb.uba.ar

\section{Introduction}

Retinoblastoma $(\mathrm{Rb})$ is the most common intraocular neoplasm of childhood with an estimated incidence of $1: 20,000$ live births. It is originated by homozygous inactivation of the tumor suppressor gene RB1 (13q14.1-2) that encodes a nuclear phosphoprotein $(\mathrm{pRB})$ with a role in the G1/S phase transition. Genetic alteration in both RB1 alleles leads to altered or null $\mathrm{pRB}$ expression that is critical for tumor development (Knudson, 1971).

In non-hereditary retinoblastoma (60\% of cases) both mutations in RB1 gene take place in a single retinoblast cell, and are therefore only present in tumor DNA. In hereditary Rb (40\%) the first mutation occurs in a germ-line and is either transmitted from an affected parent (hereditary transmitted $\mathrm{Rb}$ ) or acquired during gametogenesis or gestation (hereditary de novo $\mathrm{Rb}$ ), therefore all the tissues are expected to carry the first mutated copy of RB1. The second inactivating mutation is always somatic, present only in tumor DNA (Knudson, 1971). This gene inactivation mechanism might explain the presence of several tumor masses in hereditary $\mathrm{Rb}$, since all tissues would carry one mutated copy of the RB1 gene: a single inactivating mutation in the remaining wild-type allele, in any retinoblast cell, would be enough for tumor development. For that reason, hereditary disease is usually associated with bilateral, multifocal or early onset tumors. Non-hereditary Rb cases are commonly single tumors, usually appearing in children 2-3 years of age, since the chance of two inactivating mutations occurring in the same locus is very low. For that reason, the hereditary or non-hereditary nature of the disease might be inferred based on clinical features (Kato, 1994).

The aim of the present study was to identify genetic abnormalities in the RB1 tumor suppressor gene in retinoblastoma Argentine patients and to determine a possible genotype/phenotype correlation. 
All bilateral, multifocal or familial cases under study were classified as hereditary forms, expecting them to carry germline mutations. Patients with an early presentation of unilateral tumors were likely to be carriers of a germline mutation as well, while sporadic-unilateral tumors with late onset $(\geq 2$ years) were considered non-hereditary forms, and presumably out of germline mutation risk.

Since $30 \%$ of hereditary $\mathrm{Rb}$ are presented as sporadic unilateral tumors, the age at diagnosis is the critical data for $\mathrm{Rb}$ types discrimination.

Mutations reported to date are widely distributed along the 27 exons of the gene, as well as in the regulatory regions (Lohmann, 1999a). Both, length of the gene and diversity of mutations, make difficult their identification in each patient. We screened the entire RB1 gene for small mutations, by PCR-heteroduplex and direct sequencing, in hereditary and non-hereditary $\mathrm{Rb}$ cases, of early and late presentation. One identified mutation resulted to be an intronic polymorphism. The other identified mutations resulted to be $\mathrm{C}$ to $\mathrm{T}$ transitions with different locations in the RB1 gene. One patient with a late onset sporadic unilateral tumor had a germline mutation, this strongly argues against the hypothesis that age at diagnosis is a suitable method to discriminate patients for whom the research for constitutional mutations is appropriate.

The data obtained was remarkably useful for genetic counseling and planning of an appropriate early treatment in each family.

\section{Materials and Methods}

Patients We analyzed 21 non-related Argentine Rb patients with different clinical presentations. Six familial bilateral cases, 7 nonfamilial bilateral cases, and 8 sporadic unilateral cases. They were classified according to tumor presentation and age of onset into hereditary and non-hereditary forms (Table 1).

Department of Genetics, Ophthalmology and Oncology of several Buenos Aires Pediatric Hospitals referred the patients. Diagnosis was established using current ophthalmological and histophathological criteria.

DNA Samples Constitutional DNA was isolated from leukocytes of peripheral blood anticoagulated with EDTA 0.5\%, using CTAB method (cetyltrimethylammonium bromide), chloroform-isoamal alcohol purification and ethanol precipitation.

PCR amplification The 27 exons, as well as promoter and polyadenylation region were amplified using the primers described by Lohmann et al. (1994) including flanking intron regions. Amplification was performed in a $25 \mu \mathrm{l}$ of total volume containing $150 \mathrm{ng}$ of genomic DNA, $20 \mathrm{nmol}$ of each dNTP, 1 unit of Taq polymerase (Life Technologies, Carlsbad, USA) and $50 \mathrm{pmol}$ of each primer (Life Technologies), in standard buffer conditions, except for exon 1 where $2.5 \%$ DMSO was used. Thermal cycling (denaturing for $1 \mathrm{~min}$ at $94^{\circ} \mathrm{C}$, annealing for $1 \mathrm{~min}$ at $52^{\circ} \mathrm{C}-62^{\circ} \mathrm{C}$ and extension for $1 \mathrm{~min}$ at $72^{\circ} \mathrm{C}$ ) was performed in a Minicycler TM (MJ Research, Waltham, USA). 35 cycles were preceded by

Table 1. Patients analyzed in this study

\begin{tabular}{|c|c|c|c|c|c|}
\hline Patient & Clinical data & Familial history & Sex & $\begin{array}{l}\text { Age at diagnosis } \\
\text { (months) }\end{array}$ & Enucleation \\
\hline 118 & bilateral & Yes & M & Unknown & No \\
\hline 132 & bilateral & Yes & $\mathrm{F}$ & 22 & Left eye \\
\hline 162 & bilateral & Yes & $\mathrm{F}$ & 42 & No \\
\hline 163 & bilateral & Yes & $\mathrm{F}$ & 2 & Left eye \\
\hline 166 & bilateral & Yes & M & 47 & Left eye \\
\hline 172 & bilateral & Yes & M & 30 & Yes \\
\hline 151 & bilateral & No & M & 12 & Both eyes \\
\hline 176 & bilateral & No & $\mathrm{F}$ & 33 & Right eye \\
\hline 182 & bilateral & No & M & Unknown & Right eye \\
\hline 190 & bilateral & No & $\mathrm{F}$ & 8 & Both eyes \\
\hline 211 & bilateral & No & $\mathrm{F}$ & 16 & No \\
\hline 237 & bilateral & No & M & 3 & No \\
\hline 253 & bilateral & No & $\mathrm{F}$ & 4 & No \\
\hline 204 & unilateral & No & M & 27 & Yes \\
\hline 227 & unilateral & No & $\mathrm{F}$ & 36 & No \\
\hline 215 & unilateral & No & M & 7 & Yes \\
\hline 233 & unilateral & No & $\mathrm{F}$ & 4 & Left eye \\
\hline 44 & unilateral & No & M & 4 & Yes \\
\hline 262 & unilateral & No & $\mathrm{F}$ & 4 & Right eye \\
\hline 265 & unilateral & No & $\mathrm{F}$ & 7 & Right eye \\
\hline 274 & unilateral & No & M & 9 & Left eye \\
\hline
\end{tabular}


4 min at $94^{\circ} \mathrm{C}$ and followed by an additional $4 \mathrm{~min}$ at $72^{\circ} \mathrm{C}$.

Screening for small mutations In order to standardize conditions, heteroduplex was set up using 4 control mutated samples. To enhance heteroduplex formation samples were heated at $95^{\circ} \mathrm{C}$ for $1 \mathrm{~min}$ and slowly cooled at $40^{\circ} \mathrm{C}$ during $1 \mathrm{~h}$. The best electrophoretic resolution between homoduplex and heteroduplex bands was achieved in 9\% acrylamide-bisacrylamide $(29: 1)$ gels with $3 \%$ glycerol and $1.16 \mathrm{M}$ urea. These slightly denaturing gels seemed to improved detection of conformational changes produced by single base mismatches, increasing differential migration of heteroduplex bands. Three microliters of each PCR product were mixed with $1 \mu \mathrm{l}$ of loading buffer (30\% glycerol, $0.25 \%$ xylenecyanol, $0.25 \%$ bromophenol blue) and electrophoresed at $50 \mathrm{~V}$ over night or at $250 \mathrm{~V}$ for $3 \mathrm{~h}$ at room temperature, in $1 \mathrm{x}$ TBE (pH 8) running buffer (V16 vertical cube, $0.8 \mathrm{~mm}$ thick gel-Life Technologies). Silver staining was used to visualize DNA bands.

Mutational screening algorithm Although mutations described for the RB1 gene show no preferential distribution, we decided to analyze different regions according to the following rationale:

-Step 1: Since CGA codons are affected by $\mathrm{C}$ to $\mathrm{T}$ transition resulting from deamination of 5-methylcytosine, those areas were scanned initially, including exons $8,11,14,15,17,18,19,23,25$, and 27.

-Step 2: Exons 2, 3, and 4 with pur-pyr monotone repetitions were added.

-Step 3: Exon 13 of the A-box pocket domain and exons 20 and 21 of the B-box pocket domain were analyzed.

All the remaining exons were finally tested to screen the whole gene length. The amplicons with an altered electrophoretic pattern were analyzed by direct sequencing.

Sequence analysis PCR products were purified from unincorporated primers and dNTPs by ConcertTM ultrafiltration columns (Nucleic acid Purification System, Life-Technologies) for direct sequencing (Sanger et al., 1977). Primers were radioactively end-labeled with $\gamma^{32} \mathrm{P}[\mathrm{ATP}]$, using Fmol DNA sequencing kit (Promega, Madison, USA) according to supplier's specifications. Sequencing reaction products were electrophoresed on a $6 \%$ polyacrylamide denaturing ( $7 \mathrm{M}$ urea) gel, that was then dried and autoradiographed overnight using Kodak BioMax MR-1 films (Rochester, USA).

Restriction enzyme analysis Once a mutation was detected, we searched for a possible restriction enzyme recognition site to be created or abolished. The germ-line mutation identified in exon 18 from patient 132, created a new restriction enzyme recognition site for DdeI, by changing the sequence CCGAG to CTGAG, allowing the analysis by digestion profile. One $\mu \mathrm{g}$ of PCR product was incubated with 10 units of the DdeI restriction enzyme (Invitrogen, Carlsbad, USA) in appropriate buffer during $2 \mathrm{~h}$ at $37^{\circ} \mathrm{C}$. Fragments were electrophoretically separated in a $10 \%$ polyacrylamide $(19: 1)$ gel and visualized by silver staining. Fragments of 117 and $85 \mathrm{bp}$ were expected to be obtained for the digested mutant allele and a single undigested $202 \mathrm{bp}$ fragment for the normal one. This restriction analysis was performed in the samples from other patients as well, in order to rule out the presence of the same mutation in any of them. None of the other mutations identified were known to change a restriction enzyme recognition site.

\section{Results}

In the present study, we used a screening sequencing method to evaluate DNA germline mutations in RB1 gene in 21 unrelated retinoblastoma patients with different clinical presentation. Heteroduplex analysis was performed as prescreening method for small length alterations of the gene. To optimize detection, different gel and sample conditions, such as time, and degree of denaturation and electrophoresis running time were tested with 4 control mutated samples. Even though no hot spot for RB1 gene mutations have been reported, an initial approach was undertaken in which the gene was scanned for mutations in different specific regions. The complete coding region, promoter and polyadenylation site were subsequently analyzed.

Four germline mutations were identified in the 13 bilateral cases (two familial and two sporadic), and two germline mutations were identified in the 8 sporadic unilateral cases. One of these was found in a case of delayed onset. Overall, 6

Table 2. Single substitutions identified in this study

\begin{tabular}{|c|c|c|c|c|c|c|c|c|}
\hline Patient & Site & Description $^{\mathrm{a}}$ & $\begin{array}{l}\text { Codon } \\
\text { number }\end{array}$ & $\begin{array}{l}\text { Nucleotide } \\
\text { change }^{\mathrm{b}}\end{array}$ & $\begin{array}{c}\text { Expected } \\
\text { Consequence }\end{array}$ & $\begin{array}{l}\text { Disease } \\
\text { Type }\end{array}$ & $\begin{array}{c}\text { Age at } \\
\text { diagnosis } \\
\text { (months) }\end{array}$ & Recurrences $^{\mathrm{c}}$ \\
\hline 163 & Ex 11 & g.65386 C>T & 358 & CGA $>$ TGA & Arg $\rightarrow$ Stop & Familial Bilateral & 2 & Yes (7) \\
\hline 176 & Ex 13 & g.73835 C>T & 433 & $\mathrm{GCT}>\mathrm{GTT}$ & Ala $\rightarrow$ Val & Sporadic Bilateral & 33 & No \\
\hline 132 & Ex 18 & g.150037 C>T & 579 & CGA $>$ TGA & Arg $\rightarrow$ Stop & Familial Bilateral & 22 & Yes (12) \\
\hline 227 & Ex 18 & g.150037 C>T & 579 & CGA $>$ TGA & Arg $\rightarrow$ Stop & Sporadic Unilateral & 36 & Yes (12) \\
\hline 237 & Ex 23 & g.162237 C>T & 787 & $\mathbf{C G A}>\mathbf{T G A}$ & Arg $\rightarrow$ Stop & Sporadic Bilateral & 3 & Yes (9) \\
\hline 274 & In 25 & g.174351 T>A & IVS26-10 & TAAT $>$ AAAT & Unknown & Sporadic Unilateral & 9 & Yes (T85\%-A $15 \%)$ \\
\hline
\end{tabular}

${ }^{a}$ Mutation nomenclature after Antonarakis (1998)

${ }^{b}$ Nucleotides change in bold

'Presence of the mutation in RB database as germline mutation, number of occurrences in parenthesis 
(A)

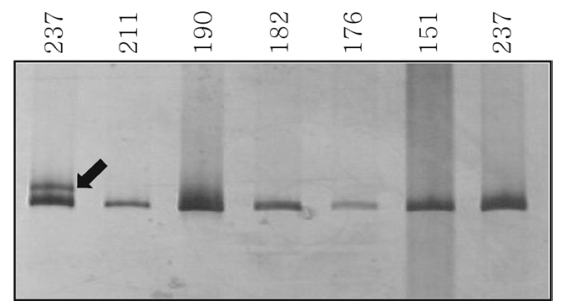

(B)

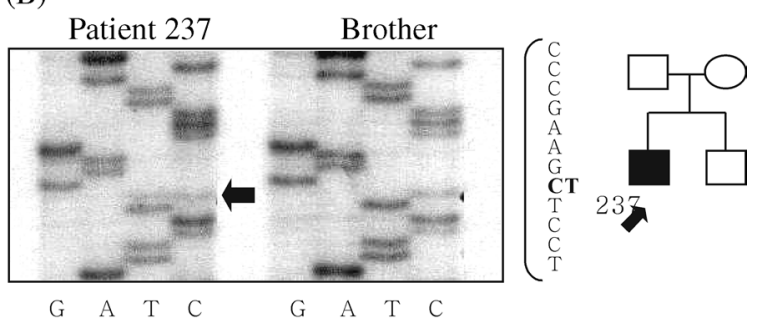

Fig. 1. Heteroduplex and sequencing analysis of exon 23. Heteroduplex was performed in a $9 \%(29: 1)$ polyacrylamide gel with $3 \%$ glycerol and $1,16 \mathrm{M}$ urea, bands were visualized by silver staining. Samples with an altered migration pattern were sequenced using $\gamma^{32} \mathrm{P}$ ended labeled forward primers. Pedigree of the patients family is shown. (a) Exon 23 heteroduplex of sporadic bilateral patients. (b) DNA sequencing of exon 23 PCR product for patient 237 (left) and his healthy brother (right). Numbers of samples are indicated in the figure. In heteroduplex, black arrow indicates altered migration pattern. In sequences, black arrow indicates position of the $\mathrm{C}$ to $\mathrm{T}$ heterozygous transition.

germline mutations were detected in the 21 ascertained patients (Table 2).

Patient 237 (sporadic bilateral tumor) PCR product from exon 23 displayed an altered migration pattern by heteroduplex analysis (Fig. 1a). Direct sequencing of this exon revealed an heterozygous $\mathrm{C}$ to $\mathrm{T}$ transition in codon 787 (162237 C>T) (Fig. 1 b), changing CGA to TGA, Arg to Stop codon (R787X), presumably resulting in a truncated protein. Parents and a brother of the patient did not show the mutation in their peripheral blood DNA by direct sequencing.

Patient 176 (sporadic bilateral tumor) Heteroduplex analysis of exon 13 PCR product showed an abnormal migration band, (Fig. 2a). Direct sequencing of this sample revealed an heterozygous $\mathrm{C}$ to $\mathrm{T}$ transition in codon 433 (73835 C>T) (Fig. 2b). The mutation converts codon GCT from an alanine to a GTT valine codon (A433V). Neither of the parents showed this mutation by direct sequencing. This alteration has not been previously reported.

Patient 163 (bilateral tumor with familial history) PCR product of exon 11 showed an abnormal heteroduplex banding pattern (Fig. 3a). Direct sequencing revealed an heterozygous $\mathrm{C}$ to $\mathrm{T}$ transition in codon 358 (65386 $\mathrm{C}>\mathrm{T})$,
(A)

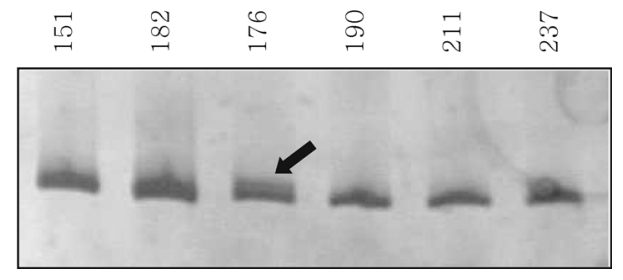

(B)

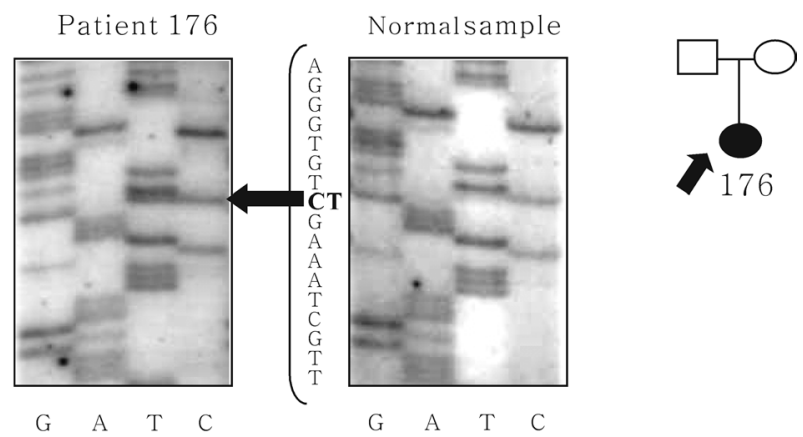

Fig. 2. Heteroduplex and sequencing analysis of exon 13. Heteroduplex was performed in a 9\% $(29: 1)$ polyacrylamide gel with $3 \%$ glycerol and $1,16 \mathrm{M}$ urea, bands were visualized by silver staining. Samples with an altered migration pattern were sequenced using $\gamma^{32} \mathrm{P}$ ended labeled forward primers. Pedigree of the patients family is shown. (a) Exon 13 heteroduplex of sporadic bilateral patients. (b) DNA sequencing of exon 13 PCR product for patient 176 (left) and a normal sample (right). Numbers of samples are indicated in the figure. In heteroduplex, black arrow indicates altered migration pattern. In sequences, black arrow indicates position of the $\mathrm{C}$ to $\mathrm{T}$ heterozygous transition.

resulting in a change of CGA (Arg) to TGA (Stop codon). This R787X change presumably produced a truncated protein. His affected mother (bilateral retinoblastoma, diagnosed at 2 years of age), showed the same mutation in their peripheral blood DNA by direct sequencing (Fig. 3b).

Patient 132 (bilateral tumor with familial history) Heteroduplex analysis of exon 18 PCR product revealed a band of abnormal migration in addition to the normal homoduplex band in the patient (Fig. 4a). Direct sequencing showed an heterozygous $\mathrm{C}$ to $\mathrm{T}$ transition in codon 579 $(150037 \mathrm{C}>\mathrm{T})$, resulting in a change of CGA to TGA, Arg to Stop codon (R579X). Similar sequencing patterns were found in the other affected members of the family (brother, bilateral $\mathrm{Rb}$ diagnosed at 2 months of age; father, healthy carrier whose mother had had unilateral retinoblastoma) (Fig. 4b). This mutation would probably produce a premature translation termination at amino acid 578, resulting in a truncated protein.

Since the mutation created a new DdeI restriction enzyme recognition site, changing the sequence CCGAG to CTGAG, exon 18 PCR product from patient 132 and her family were tested with this enzyme. The patient, her affected brother, and 
(A)

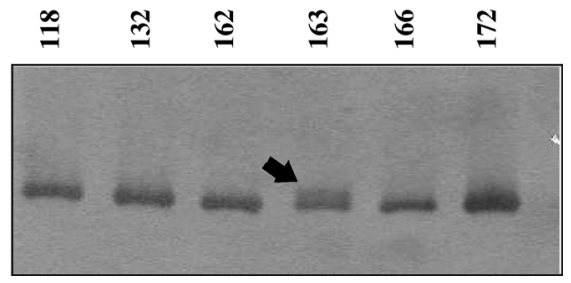

(B)

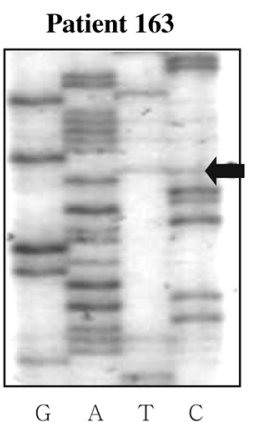

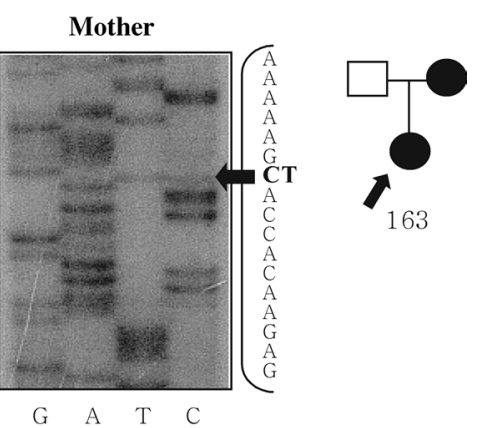

$\begin{array}{lllll}\text { G } & \text { A } & \text { T } & \text { C }\end{array}$
(A)
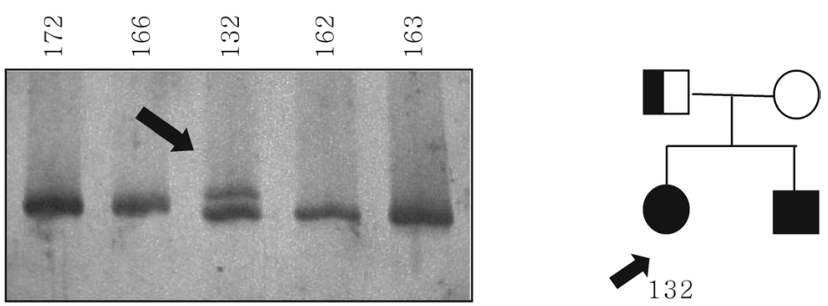

(B)

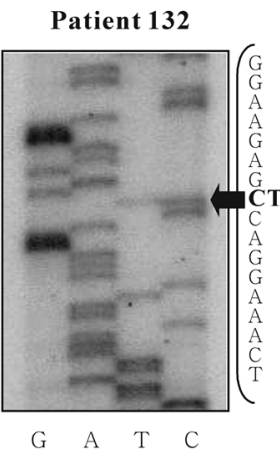

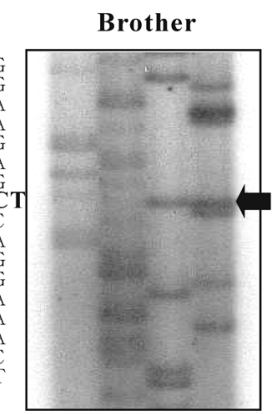

G $\quad$ A $\quad$ T $\quad$ C

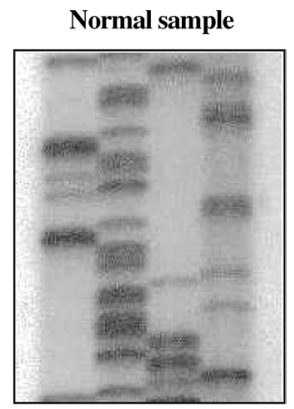

G A $\quad \mathrm{T} \quad \mathrm{C}$
Heteroduplex was performed in a $9 \%(29: 1)$ polyacrylamide gel with $3 \%$ glycerol, bands were visualized by silver staining. Samples with an altered migration pattern were sequenced using $\gamma^{32} \mathrm{P}$ ended labeled forward primers. Pedigree of the patients family is shown. (a) Exon 11 heteroduplex of familial bilateral patients. (b) DNA sequencing of exon 11 PCR product for patient 163 (left) and his affected mother (right). Numbers of samples are indicated in the figure. In heteroduplex, black arrow indicates altered migration pattern. In sequences, black arrows indicate position of the $\mathrm{C}$ to $\mathrm{T}$ heterozygous transition.

her healthy carrier father resulted in a heterozygous cleaved pattern as expected from clinical data (Fig. 5a). DdeI digestion was used as a simple method of exclusion of the same mutation in all of the other patients analyzed, one of the tested patients (227) resulted in a cleaved pattern (Fig. 5a).

Patient 227 (unilateral tumor of late onset with no familial history) In spite of being presumably a non hereditary case, because of her clinical presentation, the patient showed an heterozygous DdeI cleaving pattern for exon 18 (lane 11 of Fig. 5a). Direct sequencing of the PCR product confirmed the $\mathrm{C}$ to $\mathrm{T}$ transition in codon 579 , the same as in patient 132 . (150037C>T, R579X) (Fig. 4b). The mutation was not detected in the mother or in the brother of the patient 227. This patient presented an unilateral sporadic $\mathrm{Rb}$ of delay onset (3 years old at diagnosis), clinically characterized and treated as non-hereditary $\mathrm{Rb}$. That $\mathrm{C}$ to $\mathrm{T}$ truncated mutation has not been previously reported in non-hereditary or late onset unilateral cases.

Patient 274 (sporadic unilateral Rb) The heteroduplex pattern for exon 26 PCR products showed an additional band (Fig. 6a). Sequencing of this region revealed a heterozygous $\mathrm{T}$
Fig. 4. Heteroduplex and sequencing analysis of exon 18. Exon 18 heteroduplex of bilateral familial patients performed in a $9 \%$ (29:1) polyacrylamide gel with $3 \%$ glycerol and $1.16 \mathrm{M}$ urea, bands were visualized by silver staining. Black arrow indicates altered migration pattern. Sequencing analysis of exon 18 PCR products from patient 132 (left), his affected brother (middle) and from an unaffected individual (right). Samples were sequenced using $\gamma^{32} \mathrm{P}$ ended labeled forward primer. Black arrows indicate position of the $\mathrm{C}$ to $\mathrm{T}$ heterozygous transition. Pedigree of the patients family is shown.

to A transversion located 10 bases upstream of the 5 exon 26 splicing site (IVS25-10T>A or g.174351 T>A). This corresponded to the allelotype at the RB1.26 sequence polymorphism (Fig. 6b).

Patients 172, 253, 262, 265, 274, 297 A thymidine was found at position g. 174438 where, according to the original published sequence, a cytosine should have been present. This non concordant $\mathrm{T}$ base was identified in intron 26 (IVS26 + 28). It is worthy of notice the homozygous T status in the six samples. It remains to be established whether this base change represents a new polymorphism (Fig. 6b).

\section{Discussion}

In the present study of 21 retinoblastoma families, 6 mutations in the RB1 gene were detected (29\%), four nonsense mutations, one missense and one intronic polymorphism. The five exonic alterations were $\mathrm{C}$ to $\mathrm{T}$ transitions in $\mathrm{CpG}$ dinucleotides, which are the most frequently observed single base pair substitutions causing human genetic diseases (Mancini, 1997), the sixth was a T to A intronic transversion, 
(A)

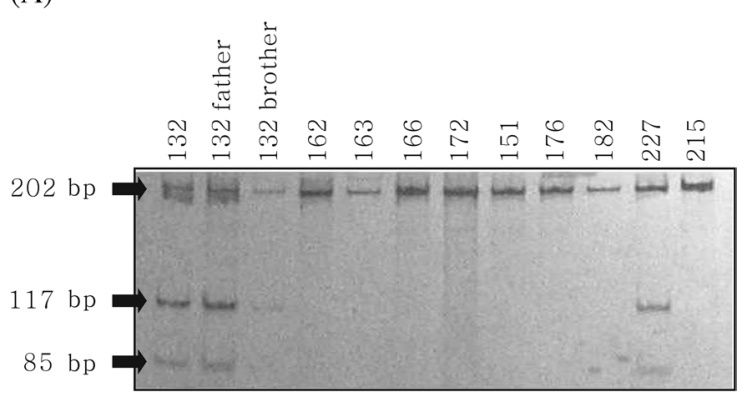

(B)

\section{Patient 227}

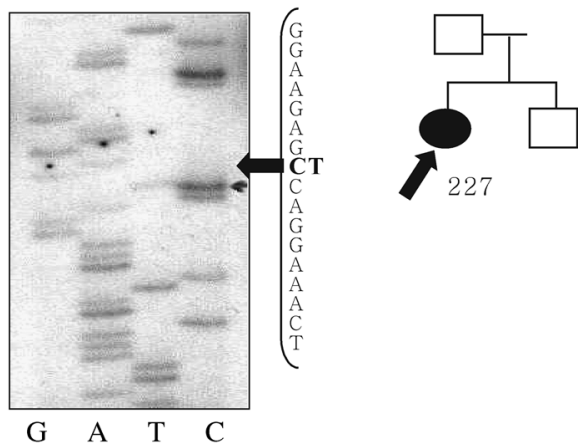

Fig. 5. Heteroduplex, sequence and restriction enzyme analysis. The germ-line mutation identified in exon 18 of patient 132, created a new restriction enzyme recognition site for DdeI, by changing the sequence CCGAG to CTGAG. The mutated DNA is resolved in two bands electrophoretically separated in a $10 \%$ polyacrylamide (19:1) silver stained gel; the wild-type DNA generates one undigested band. (molecular weight on the left of the figure). Lanes 1 to 12 , patients of several presentations. Lanes 1, 2, 3, and 11 showed a heterozygous digested pattern. Lane 1: bilateral familial patient; lane 2: healthy carrier father; lane 3: affected brother; lane 11: sporadic unilateral case. (b) DNA sequencing of exon 18 PCR product for patient 227. The $\mathrm{C}$ to $\mathrm{T}$ transition is marked with a black arrow. Pedigree for 227 patient family is shown. Numbers of samples are indicated in the figures.

with an expected harmless consequence in the translated protein. Four of the five exonic mutations produced nonsense alterations, changing the arginine CGA codon to TGA stop codon, a very common finding reported for different CGA codons in the RB1 gene (Mancini, 1997). These mutations were found in exon 11 (case 163), exon 18 (cases 132, 227) and exon 23 (case 237), therefore the translated proteins would be, most probably, too short to be functional, loosing fundamental domains for their activity. The fifth exonic mutation was novel, and replaced an alanine 433 codon (GCT) in exon 13 with a valine GTT codon (case 176). As the exon 13 is included in box-A of the $\mathrm{Rb}$ protein pocket domain, this missense alteration may have a causal effect in the loss of RB function.

Four of the mutations identified by us were located in CGA codons, which were the first step of analysis in our schedule,
(A)

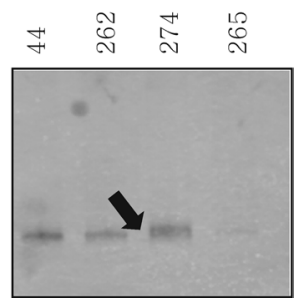

(B)

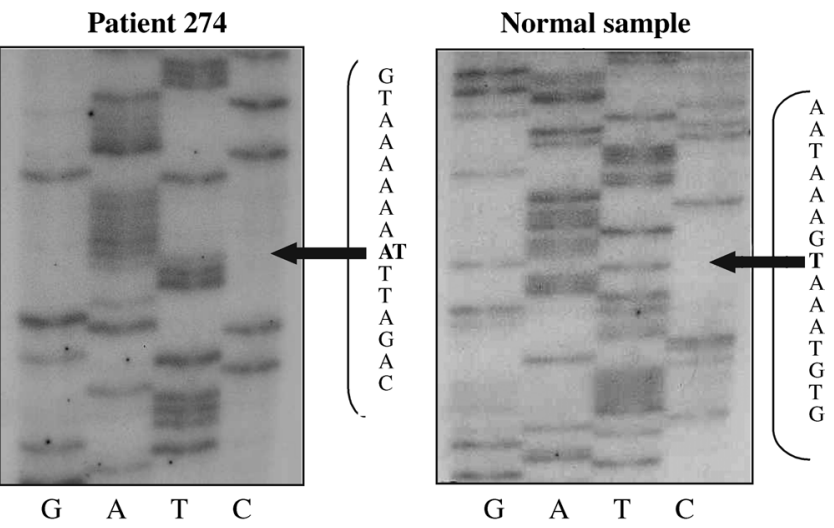

Fig. 6. Heteroduplex and sequence analysis of exon 26. Heteroduplex was performed in a 9\% (29:1) polyacrylamide gel with $3 \%$ glycerol and $1,16 \mathrm{M}$ urea; bands were visualized by silver staining. Samples with an altered migration pattern were sequenced using $\gamma^{32} \mathrm{P}$ ended labeled forward primers. (a) Exon 26 heteroduplex of sporadic unilateral patients. Altered migration patterns is observed in patient 274 (marked with a black arrow). Numbers of samples are indicated in the figures. (b) DNA sequencing of 26 exon PCR product for patient 274. Left, T to A transversion is observed in the intronic sequence, 10 bases upstream of exon 26, a double banding pattern is observed (marked with a black arrow), reverse primer was used. Right, an homozygous $\mathrm{T}$ base was detected 28 bases downstream of exon 26. According to published sequence a $\mathrm{C}$ base is expected at that position.

proving to be a practical manner of time profit in the search. Three of the five RB1 gene mutations $(60 \%)$ identified in this study (patients 176, 227, 237) were de novo, in accordance with the published epidemiological data. Two mutations were familial and the other one was a polymorphic site, its distribution in our population remains to be determined.

Comparing with Lohmann database (Lohmann, 1999b) and published data, mutation A433V in exon 13 (case 176) has not been previously reported. Beside this novel mutation, only two others have been reported for exon 13, one nonsense mutation Q413X and one missense in splicing recognition site, both in unilateral cases (Klutz et al., 1999; Lohmann et al., 1997). Missense substitutions at alanine codons are rare events in retinoblastoma, with only one report case in exon 19 , A635P (Blanquet et al., 1995). Mutation R787X in exon 23 (case 237) has been reported as germline in 8 bilateral and 1 unilateral cases, mutation R358X in exon 11 (patient 163) has 
been reported as germline in 6 bilateral and 2 unilateral cases and mutation R579X in exon 18 (patient 132 and 227) has been reported as germline in 11 bilateral cases. The $\mathrm{T}$ to $\mathrm{A}$ alteration in intron 25 sequence (patient 274) corresponded to a polymorphism reported by Yandell and Dryja (1989) and founded in three other patients by Horsthemke (1996). A non concordant $\mathrm{T}$ base, according to published sequence, was identified in intron 26 (IVS26 + 28) in six samples (patients $172,253,262,265,274$, and 297). The homozygous T status of the six of them makes it interesting for revealing whether it is a possible mistake in the original sequencing or a polymorphism.

Analyzing the familial cases, the $\mathrm{C}$ to $\mathrm{T}$ truncated mutation (R579X) of patient 132, was also found in his affected brother and in his healthy father (with previous Rb history). It is noteworthy that the father remained healthy in spite of the presumably truncated effect of this germline mutation and of the expected full penetrance produced by this mutation (Harbour, 2001). Meanwhile, the $\mathrm{C}$ to $\mathrm{T}$ truncated mutation in patient 163 (R358X), was found in his affected father, who also developed a bilateral retinoblastoma (2 years of age). The truncated mutation in exon 18 (R579X), was found in two patients of different clinical presentation, one bilateral familial case (patient 132) and one unilateral sporadic of late onset (patient 227). This mutation has been previously reported as a germline mutation in patients with bilateral tumors (Szijan, 1995; Lohmann mutation database, 1999), but has never been described in a late onset sporadic unilateral form in peripheral blood. It is highly remarkable that the same germline mutation is presumed to be responsible for so different phenotypes, several members of the family affected with bilateral tumors in one case and only one tumor at a really late onset present in the other case.

According to some authors low penetrance of the mutation (unilateral, unifocal tumors) is based on compromising gene function rather than abolishing it (Dryja, 1993; Ohtani-Fujita et al., 1997; Zajaczek, 1999; Alonso, 2001; Harbour, 2001). These mutations would be mainly promoter alterations and missense mutations or in-frame deletions, which would not lead to creation of stop codons. Our results are not in accordance with those findings since a truncated mutation was found in a low penetrance retinoblastoma. This disagreement might be explained through:

As no tumor sample was available, chromosomal mechanisms occurring as second somatic mutational event could not be studied. If the second mutation affecting the other allele was "weak or benign" (leaving functional $\mathrm{Rb}$ protein), that would explain low penetrance of the disease in patient 227. For instance, hypermethylation of 5 ' regulatory region in RB1 gene, would produce a quantitative decrease in the expression of the normal RB protein, those alleles would show structurally normal but aberrantly methylated (Greger, 1994; Zeschnigk, 1999). On the contrary, the second mutational event in patient 132 (full penetrance, early onset $\mathrm{Rb}$ ) could involve a mitotic recombination or non-disjunction chromosomal event that would leave no functional $\mathrm{Rb}$ protein for cell cycle arrest.

Some authors described a variable expressivity or less tumor development in $\mathrm{Rb}$ patients by mutational mosaicism. According to that model, reduced expressivity reflects the fewer cells that are target for a second mutation. (Sipper et al., 1998)

In addition to this, the age at diagnosis could be biased because of familial history, prompting an early ophthalmological examination in the offspring, revealing tumors at initial steps of evolution. Otherwise, there are no regular exams in general population.

In conclusion the age at diagnosis might not be an accurate indicator for the age of onset of the disease, since expectation could accelerate the time at diagnosis. Is it possible that a late onset could be related either with an early development of the disease with a slow progression or with a late clinical diagnosis? Probably more factors other than the mutation itself could be interacting for early or late tumor development, as immunological factors, modulator genes or other complex genetic abnormalities that could be interacting in neoplasia initiation or progression (Harbour, 2001; Herzog et al., 2001). The clinical importance of the mutation analysis is clearly demonstrated in this study, where a reliable identification allowed assigning a new risk prediction rate to patient relatives. In addition, the study of sporadic unilateral retinoblastoma seems to be important and of potential value. To perform an efficient detection method of mutations in RB1 gene, the knowledge of the gene organization and its mutational spectrum is fundamental. A higher number of mutations must be located and a saturated database must be achieved, in order to predict and locate mutational mechanisms. The development of sensitive and reliable tests as well as practical in time and certainty is a challenge to go.

Acknowledgments We thank Dr Abdala Mirta, Dr Francipane Liliana, Dr Fandiño Adriana, Dr Davila María and Dr Chantada Guillermo, for providing samples from retinoblastoma patients. This work was supported by grants from Universidad de Buenos Aires (B069) and Agencia de Promoción de Investigaciones Científicas y Tecnológicas (BID 1201/OC-AR-PICT 5-8684).

\section{References}

Alonso, J. (2001) Spectrum of germline RB1 gene mutations in Spanish retinoblastoma patients: phenotypic and molecular epidemiological implications. Hum. Mutat. 17, 412-422.

Blanquet, V., Turleau, C., Gross-Morand, M. S., SenamaudBeaufort, C., Doz, F. and Besmond, C. (1995) Spectrum of germline mutations in the RB1 gene: a study of 232 patients with hereditary and non hereditary retinoblastoma. Hum. Mol. Genet. 4, 383-388.

Dryja, T. (1993) Molecular Etiology of low-penetrance retinoblastoma in two pedigrees. Am. J. Hum. Genet. 52, 1122- 
1128.

Greger, V., Debus, N., Lohmann, D., Hopping, W., Passarge, E. and Horsthemke, B. (1994) Frequency and parental origin of hypermethylated RB1 gene alleles in retinoblastoma. Hum. Genet. 94, 491-496.

Harbour, J. (2001) Molecular basis of low-penetrance retinoblastoma. Arch. Ophthalmol. 119, 1699-1704.

Herzog, S., Lohmann, D. R., Buiting, K., Schuler, A., Horsthemke, B., Rehder, H. and Rieder, H. (2001) Marked differences in unilateral isolated retinoblastomas from young and older children studied by comparative genomic hybridization. Hum. Genet. 108, 98-104.

Kato, M. (1994) Parental origin of germ-line and somatic mutations in the retinoblastoma gene. Hum. Genet. 94, 31-38.

Klutz, M., Horsthemke, B. and Lohmann, D. R. (1999) RB1 gene mutations in peripheral blood DNA of patients with isolated unilateral retinoblastoma. Am. J. Hum. Genet. 64, 667-668.

Knudson, A. G. (1971) Mutation and cancer: statistical study of retinoblastoma. Proc. Natl. Acad. Sci. USA 68, 820-823.

Lohmann, D. R. (1999a) RB1 gene mutations in retinoblastoma. Hum. Mutat. 14, 283-288.

Lohmann, D. R. (1999b) RB1 mutation database (www.dlohmann.de/Rb/mutations.html)

Lohmann, D. R., Brandt, B., Hopping, W., Passarge, E. and Horsthemke, B. (1994) Distinct RB1 gene mutations with low penetrance in hereditary retinoblastoma. Hum. Genet. 94, 349354.

Lohmann, D. R., Gerick, M., Brandt, B., Oelschlager, U., Lorenz,
B., Passarge, E. and Horsthemke, B. (1997) Constitutional RB1-gene mutations in patients with isolated unilateral retinoblastoma. Am. J. Hum. Genet. 61, 282-294.

Mancini, D. (1997) Constitutively methylated CpG dinucleotides as mutation hot spots in the retinoblastoma gene (RB1). Am. J. Hum. Genet. 61, 80-87.

Ohtani-Fujita, N., Dryja, T. P., Rapaport, J. M., Fujita, T., Matsumura, S., Ozasa, K., Watanabe, Y., Hayashi, K., Maeda, K., Kinoshita, S., Matsumura, T., Ohnishi, Y., Hotta, Y., Takahashi, R., Kato, M. V., Ishizaki, K., Sasaki, M. S., Horsthemke, B., Minoda, K. and Sakai, T. (1997) Hypermethylation in the retinoblastoma gene is associated with unilateral, sporadic retinoblastoma. Cancer Genet. Cytogenet. 98, 43-49.

Sippel, K. C., Fraiol, R. E., Smith, G. D., Schalkoff, M. E., Sutherland, J., Gallie, B. L. and Dryja, T. P. (1998) Frequency of somatic and germ-line mosaicism in retinoblastoma: implications for genetic counseling. Am. J. Hum. Genet. 62, 610-619.

Szijan, I. (1995) Identification of RB1 germ-line mutations in Argentinian families with sporadic bilateral retinoblastoma. $J$. Med. Genet. 32, 475-479.

Zajaczek, S. (1999) Frequency and nature of germ-line RB1 gene mutations in series of patients with sporadic unilateral retinoblastoma. Eur. J. Cancer 35, N13, 824-827.

Zeschnigk, M. (1999) A PCR test for detection of hypermethylated alleles at the retinoblastoma locus. J. Med. Genet. 36, 793-794. 